\title{
Pathophysiology of fluid imbalance
} Uwe Kreimeier

\author{
Ludwig-Maximilians-Universität München, Munich, Germany
}

Received: 3 August 2000

Published: 13 October 2000
Crit Care 2000, 4 (suppl 2):S3-S7

(c) Current Science Ltd (Print ISSN 1364-8535; Online ISSN 1466-609X)

\begin{abstract}
Fluid imbalance can arise due to hypovolemia, normovolemia with maldistribution of fluid, and hypervolemia. Trauma is among the most frequent causes of hypovolemia, with its often profuse attendant blood loss. Another common cause is dehydration, which primarily entails loss of plasma rather than whole blood. The consequences of hypovolemia include reduction in circulating blood volume, lower venous return and, in profound cases, arterial hypotension. Myocardial failure may result from increased myocardial oxygen demand in conjunction with reduced tissue perfusion. Finally, anerobic metabolism due to reduced perfusion may produce acidosis and, together with myocardial dysfunction, precipitate multi-organ failure. The splanchnic organs are particularly susceptible to the deleterious effects of hypotension and hypovolemic shock, and these effects, depending upon their duration and severity, may be irreversible despite restoration of normovolemia by fluid administration. Patient monitoring in the intensive care unit typically relies upon central venous pressure devices, whereas the primary focus in the operating theater is blood volume deficit estimated from suction devices. However, estimates of intraoperative blood loss can be inaccurate, potentially leading to inappropriate fluid management. Normovolemia with maldistribution of fluid can be encountered in shock-specific microcirculatory disorders secondary to hypovolemia, as well as pain and stress. Consequent vasoconstriction and reduced tissue driving pressure, as well as leukocyte and platelet adhesion, and liberation of humoral and cellular mediators, may impair or abolish blood flow in certain areas. The localized perfusion deficit may contribute to multi-organ failure. Choice of resuscitation fluid may be important in this context, since some evidence suggests that at least certain colloids might be helpful in diminishing post-ischemic microvascular leukocyte adherence. Excessive volume administration may lead to fluid overload and associated impairment of pulmonary function. However, entry of fluid into the lungs may also be facilitated by increased vascular permeability in certain pathologic conditions, especially sepsis and endotoxemia, even in the absence of substantially rising hydrostatic pressure. Another condition associated with elevated vascular permeability is systemic capillary leak syndrome. The chief goal of fluid management, based upon current understanding of the pathophysiology of fluid imbalance, should be to ensure adequate oxygen delivery by optimizing blood oxygenation, perfusion pressure, and circulating volume.
\end{abstract}

Keywords: capillary permeability, hydrostatic pressure, hypotension, osmotic pressure, shock

$\mathrm{Gl}=$ gastrointestinal; $\mathrm{HA}-1 \mathrm{~A}=$ human monoclonal antibody against the lipid A moiety of bacterial lipopolysaccharide; MAP = mean arterial blood pressure; $\mathrm{pHi}=$ gastric intramucosal $\mathrm{pH} ; \mathrm{SCLS}=$ systemic capillary leak syndrome. 


\section{Introduction}

A discussion of the pathophysiology of fluid imbalance typically focuses on hypovolemia. A relevant clinical scenario is described in the following case report.

\section{Case presentation}

A laboratory worker was taken to the emergency department with malaise, headache, nausea and vomiting [1]. There was evidence of circulatory failure and infection. The patient's pulse was 114 beats $/ \mathrm{min}$, blood pressure $42 / 20 \mathrm{mmHg}$ and oral temperature $40^{\circ} \mathrm{C}$. The pre-load filling pressure was very low, while cardiac index was elevated. Clinical symptoms were consistent with a generalized capillary leak syndrome. The patient received 14.9 I fluid in excess of measured output during the period of hypotension. Inotropic support with dopamine and norepinephrine was also provided. The presumptive diagnosis was septic shock.

It was discovered $11 \mathrm{~h}$ after admission that the patient had self-administered $1 \mathrm{mg}$ Salmonella minnesota endotoxin $2.5 \mathrm{~h}$ prior to arrival at the emergency department in an attempt to treat a recently diagnosed tumor. This single, large intravenous dose of endotoxin had provoked the manifestations of septic shock syndrome, including hypotension with high cardiac output, disseminated intravascular coagulation, abnormalities of hepatic and renal function, and pulmonary edema. A dose of $100 \mathrm{mg}$ human monoclonal antibody against the lipid $A$ moiety of bacterial lipopolysaccharide (HA-1A) antibody was administered $23 \mathrm{~h}$ after the injection of endotoxin. Norepinephrine infusion was discontinued $50 \mathrm{~h}$ after the endotoxin injection. The patient was discharged on the eighth hospital day.

Although the precipitating cause of this patient's condition was unusual, hypovolemia necessitating fluid administration is a common occurrence. Nevertheless, a proper consideration of fluid imbalance extends beyond hypovolemia alone. Fluid imbalance indeed encompasses a triad of conditions consisting of hypovolemia, normovolemia with maldistribution of fluid, and hypervolemia. Each of these is now discussed.

\section{Hypovolemia \\ Trauma}

One frequent cause of hypovolemia is trauma. In Germany, for example, trauma is still the leading cause of death among persons aged under 45 years, accounting for a third of all deaths in this age range (Statistisches Bundesamt, Wiesbaden, 1998). Estimated blood loss of $100-800 \mathrm{ml}$ has been reported in association with traumatic fracture of the humerus, $50-400 \mathrm{ml}$ with that of the forearm, $500-5000 \mathrm{ml}$ with that of the pelvis, $300-2000 \mathrm{ml}$ with that of the femur and $100-1000 \mathrm{ml}$ with traumatic fracture of the tibia [2]. Significant hypo- volemia requiring treatment can thus develop very early after trauma.

\section{Dehydration}

Another common source of hypovolemia is dehydration. Precipitating factors include: diarrhea; vomiting; ileus; gastrointestinal (Gl) fistula; gastric tube; fever; hyperglycemia and azetonuria with diabetes mellitus; and renal dysfunction (polyuria, etc.). Lost fluid in this type of patient will be comprised primarily of plasma rather than whole blood as in the trauma patient.

\section{Consequences of hypovolemia}

Reduction in circulating blood volume leads to lower venous return irrespective of its cause and, when hypovolemia is sufficiently severe, arterial hypotension [3]. Compensatory systemic release of catecholamines promotes peripheral vasoconstriction, increased cardiac contractility and tachycardia. Systemic blood pressure may therefore remain stable in the face of continuing hypovolemia. Tachycardia promotes increased myocardial oxygen demand that, in conjunction with reduced tissue perfusion, may result in myocardial failure. Finally, anerobic metabolism occurring in response to reduced perfusion may produce acidosis and, together with myocardial dysfunction, contribute to multi-organ failure.

Up to $10 \%$ of the total blood volume can be lost without affecting either cardiac output or arterial pressure. Greater than $10 \%$ loss diminishes cardiac output due to decreased preload, and oxygen delivery to the tissues falls. Arterial pressure also declines with more than $20 \%$ loss of total blood volume.

Hypovolemia and hypotension exert differential effects on organ function. In the splanchnic organs, $\alpha$-adrenergic activity is relatively high [4], and the splanchnic region is highly vulnerable in patients with hypovolemic shock and hypotension. Ischemia develops with diminished Gl perfusion, especially in the mucosal layer of the gut (Fig. 1). Mucosal integrity may be compromised. Impaired gut barrier function may allow translocation of bacteria and endotoxins, precipitating a systemic inflammatory response as a consequence of shock. In cases of severe hypovolemia, due for instance to trauma, these pathophysiologic processes may ultimately lead to the development of sepsis.

Furthermore, splanchnic organs may prove refractory to the effects of administered fluids. In a canine model of hemorrhagic shock, mean arterial blood pressure (MAP) was reduced to $40-45 \mathrm{mmHg}$ for $30 \mathrm{~min}$ [5]. Thereafter, autologous blood and saline were infused to restore MAP to preshock levels. Gastric intramucosal $\mathrm{pH}(\mathrm{pHi})$ declined sharply during shock. The $\mathrm{pHi}$ initially rebounded only partially after resuscitation, and subsequently deteriorated progressively over the remainder of the study 
follow-up period. The MAP and pHi data suggest persistent splanchnic hypoperfusion or reperfusion injury despite restoration of normovolemia by fluid administration.

\section{Monitoring hypovolemia}

The circulatory status of trauma patients is typically monitored in the prehospital phase of emergency by determinations of heart rate and systolic blood pressure. Central venous pressure devices or even a Swan-Ganz catheter may be inserted while in the intensive care unit.

The focus in the operating theater is frequently upon blood volume deficit, as estimated by blood absorbed on surgical sponges or suctioned during the procedure. Such estimates can unfortunately be inaccurate. Blood loss measured by red cell volume using nonradioactive marker sodium fluorescein was compared with that estimated intraoperatively in 30 patients undergoing gynecological operations [6]. Estimated blood loss averaged nearly $300 \mathrm{ml}$ less than measured blood loss. Blood loss in individual patients was underestimated by almost $1000 \mathrm{ml}$, and blood loss in other patients was overestimated. Thus, to the extent that fluid administration decisions are guided by estimated intraoperative blood loss, the volume infused may prove to be either insufficient or excessive.

\section{Normovolemia with maldistribution of fluid}

Shock-specific microcirculatory disorder may occur secondary to hypovolemia, and also to pain and stress. A sympathicoadrenergic reaction may provoke vasoconstriction, resulting in depressed tissue driving pressure. Flow may be attenuated or abolished in some areas as a consequence of low driving pressure, as well as leukocyte and platelet adhesion to endothelial cells and liberation of humoral and cellular mediators. These pathophysiologic processes may culminate, in some tissue areas, in a 'no-reflow/reflow paradox'. Notwithstanding apparent normovolemia and the re-establishment of normal blood pressure and cardiac output after appropriate therapeutic interventions such as fluid administration, localized perfusion may thus be inadequate or nonexistent, and multi-organ failure may ensue.

Choice of resuscitation fluid may affect at least some of the pathophysiologic processes associated with shockspecific microcirculatory disorder. In the hamster dorsal skin fold chamber model of pressure-induced ischemia, postischemic microvascular leukocyte adherence was significantly lower in animals receiving synthetic colloid (dextran) compared with crystalloid (saline) [7].

In addition to alterations in cell adhesion, activation of granulocytes may result from trauma, hypovolemia and low flow. Migrating neutrophil granulocytes have been shown to swell by $35-60 \%$ [8]. Migration was enhanced by hypo-osmolar swelling of granulocytes but inhibited by hyperosmolar shrinking. Hence, migration-associated
Figure 1

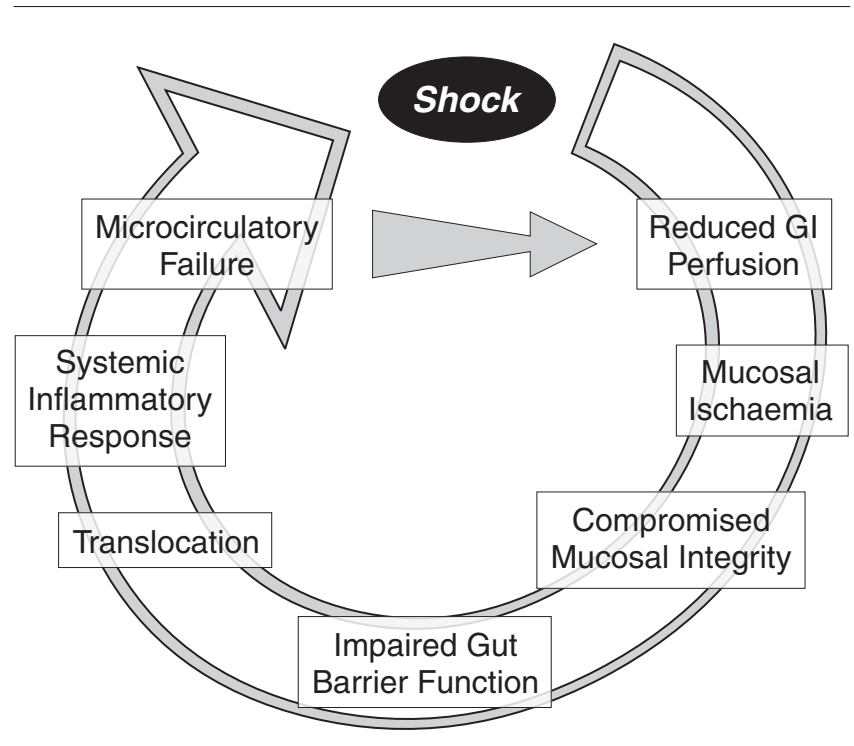

Cascade of pathophysiologic processes triggered by shock.

volume increase may be essential in allowing granulocytes to serve as the first line of defense at sites of infection. These findings also suggest the possibility of modifying granulocyte migration by therapeutic manipulation of plasma osmolality.

Cellular swelling during hypovolemic shock may also occur in endothelial cells. Endothelial swelling may be manifested, for example, in perivascular edema of the lung. This edema may contribute to pulmonary dysfunction due to the increased diffusion distance for oxygen.

\section{Hypervolemia}

Fluid overload is often viewed as primarily a phenomenon of the plasma space, but other fluid compartments may also be involved. The plasma and red blood cells together comprise a total blood volume of approximately $5 \mathrm{I}$. Both the extracellular space, occupying $15 \mathrm{I}$, and the intracellular volume of 25 I far exceed the intravascular compartment in size. When a typical volume of $500 \mathrm{ml}$ saline or albumin, for example, is infused into the circulation, all three fluid compartments may be affected.

Fluid flux between the intravascular and extravascular compartments is subject to Starling's equation. Positive capillary hydrostatic pressure combined with negative interstitial hydrostatic pressure favors extravasation of fluid (Fig. 2). The colloid oncotic pressure gradient between the intravascular and extravascular compartments opposes this hydrostatic pressure, which promotes influx of fluid into the vasculature. The arterioles, according to the traditional view, were sites of substantial fluid filtration, while fluid resorption was thought to take place in the 


\section{Figure 2}

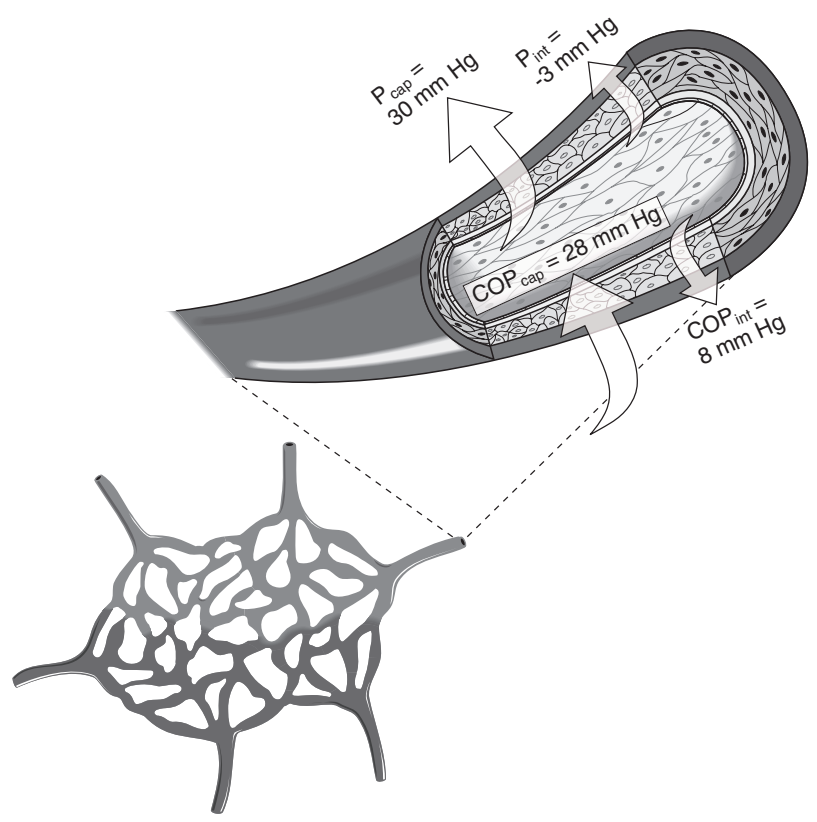

Hydrostatic and oncotic forces influencing apportionment of fluid between the intravascular and extravascular compartments, including capillary hydrostatic pressure $\left(P_{\text {cap }}\right)$, interstitial hydrostatic pressure $\left(P_{\text {int }}\right)$, capillary colloid oncotic pressure $\left(\mathrm{COP}_{\text {cap }}\right)$, and interstitial colloid oncotic pressure $\left(\mathrm{COP}_{\text {int }}\right)$.

venules. Based upon current understanding, however, this concept remains valid in the small capillaries but, except in the Gl tract and kidneys, not in the small venules where hydrostatic pressure is very low and colloid oncotic pressure altered. There is net fluid movement in the venules from the intravascular to the perivascular compartment.

A prominent concern during resuscitation of the hypovolemic polytrauma patient, for instance, is of fluid overload leading to pulmonary edema. Administration of excessive fluid, however, may not be the only contributing factor. Certain disease states, especially sepsis and endotoxemia, may per se entail increased fluid efflux from the intravascular space. Endotoxin infusion in an ovine model increased lung lymph flow five-fold with negligible increase in lung microvascular pressure [9]. A rise in lung microvascular pressure greater than two-fold was required in the absence of administered endotoxin to increase lung lymph flow two-fold. It thus remains clear, without regard to the extent of vascular permeability, that administration of sufficiently large volumes can in itself result in fluid overload to tissues, and the lung and gut are primarily affected.

Another pathologic condition associated with heightened vascular permeability is systemic capillary leak syndrome
(SCLS), also termed Clarkson's disease [10]. This condition is characterized by episodes of increased vascular permeability with extravasation of both fluid and plasma protein. SCLS patients develop hypovolemia and hypotension, and serum albumin concentration declines. Fluid extravasation leads to hematocrit increases of $70 \%$ or more, thus placing the patient at risk for thrombosis. Another feature of SCLS is secondary hyperaldosteronism, and the condition is also associated with monoclonal gammopathy. SCLS is life-threatening, and its pathogenesis remains obscure, but it is not, however, associated with infection.

One implication of Starling's equation is that hydrostatic pressure provides a primary driving force promoting fluid efflux from the capillaries. The clinician might endeavor to maintain lower hydrostatic pressure to modulate this efflux, at least for the polytrauma patient. This 'permissive hypotension' approach, however, needs to take into account the effects of low driving pressure due to low blood pressure. Neutrophil adhesion to the vessel wall has been shown to be dependent upon the level of hydrodynamic shear stress force [11]. Hence, permissive hypotension might, depending upon the extent of reduced blood pressure, lead to a clinically undesirable enhancement of neutrophil adhesion.

\section{Conclusion}

What precepts of fluid management emerge from current understanding of the pathophysiology of fluid imbalance? The fundamental requirement of the patient is oxygen, and sufficient oxygen delivery is a paramount goal of fluid management that can be attained by ensuring adequate pulmonary function. Another important goal is satisfactory perfusion pressure, which depends upon myocardial performance and vascular tone. Most importantly, however, adequate circulating volume must be maintained, and for this purpose the intravascular compartment needs to be the chief focus of fluid management.

\section{Commentary}

Jean-Louis Vincent, MD, PhD: How reliable are the currently available tools, heart rate, blood pressure and so forth, for evaluating blood volume in acutely ill patients? Some studies have employed iodine-labeled albumin. Would this approach be accurate in patients with some form of capillary leak syndrome involving albumin extravasation from the capillaries?

Uwe Kreimeier, MD: Blood pressure is affected only in the presence of hypovolemia exceeding $20 \%$ of normal blood volume. Heart rate may be increased due to pain and stress, eg in trauma patients. Intrathoracic blood volume measurement was introduced several years ago into clinical practice. For investigational purposes, labeling of erythrocytes, plasma or albumin can be useful. These 
methods allow plasma volume to be exactly calculated; however, they are not applicable to routine clinical management of the critically ill patient. Microvascular perfusion can be evaluated, and extravasation of fluid can be assessed by mercury plethysmography. This tool is highly sensitive and can be helpful in the intensive care setting. One limitation is that the patient must not be moved and must remain hemodynamically stable for at least $15 \mathrm{~min}$; thus, it is not well suited for use in the operating theater.

Andrew R Webb, MD: In the dog experiments of Oud and Kruse [5], pHi failed to recover after severe hemorrhage resulting in reduction of MAP to $40-45 \mathrm{~mm}$. A similar study has been conducted in human volunteers [12]. After a $25 \%$ drop in blood volume, pHi promptly decreased. Thereafter, stroke volume and then blood pressure fell. Upon retransfusion, $\mathrm{pHi}$ was the first of these variables to recover. The period of hypovolemia in these volunteers was relatively short, however.

Uwe Kreimeier, MD: It appears that a key consideration is the time interval during which low blood pressure or shock persists. Also, from the standpoint of the microcirculation, the degree of hypotension and hypovolemia is an important determinant of organ function and, ultimately, outcome. It should also be acknowledged that an animal model may not completely replicate the response observed in the clinical setting.

\section{References}

1. Taveira da Silva AM, Kaulbach HC, Chuidian FS, et al: Brief report: shock and multiple-organ dysfunction after self-administration of Salmonella endotoxin. N Engl J Med 1993, 328:1457-1460.

2. Burri C, Beck H, Ecke H, et al (Eds): Unfallchirurgie, edn 3. Berlin: Springer Verlag, 1982.

3. Baskett PJ: ABC of major trauma. Management of hypovolaemic shock. BMJ 1990, 300:1453-1457.

4. Meßmer K: Intestinal factors in shock: intestinal circulation. Langenbecks Arch Chir 1967, 319:890-909.

5. Oud L, Kruse JA: Progressive gastric intramucosal acidosis follows resuscitation from hemorrhagic shock. Shock 1996, 6:61-65.

6. Orth $\mathrm{VH}$, Rehm M, Thiel M, et al: First clinical implications of perioperative red cell volume measurement with a nonradioactive marker (sodium fluorescein). Anesth Analg 1998, 87:1234-1238.

7. Steinbauer M, Harris AG, Leiderer R, Abels C, Meßmer K: Impact of dextran on microvascular disturbances and tissue injury following ischemia/reperfusion in striated muscle. Shock 1998, 9:345-351.

8. Rosengren S, Henson PM, Worthen GS: Migration-associated volume changes in neutrophils facilitate the migratory process in vitro. Am J Physiol 1994, 267:C1623-C1632.

9. Brigham KL, Bowers R, Haynes J: Increased sheep lung vascular permeability caused by Escherichia coli endotoxin. Circ Res 1979, 45:292-297.

10. Clarkson B, Thompson D, Horwith M, Luckey HE: Cyclical edema and shock due to increased capillary permeability. Am J Med 1960, 29:193-216.

11. Finger EB, Puri KD, Alon R, et al: Adhesion through L-selectin requires a threshold hydrodynamic shear. Nature 1996, 379:266-269.

12. Hamilton-Davies C, Mythen MG, Salmon JB, et al: Comparison of commonly used clinical indicators of hypovolaemia with gastrointestinal tonometry. Intensive Care Med 1997, 23:276-281.
Author affiliation: Department of Anesthesiology, Ludwig-MaximiliansUniversität München, Munich, Germany

Correspondence: Uwe Kreimeier, MD, Department of Anesthesiology, Ludwig-Maximilians-Universität München, Klinikum Großhadern, Marchioninistraße 15, D-81377 Munich, Germany.

Tel: +49 897095 6412; fax: +49 897450 2817;

e-mail: kreimeier@ana.med.uni-muenchen.de 